\title{
Lucky Labeling and Proper Lucky Labeling for Bloom Graph
}

\author{
Chiranjilal Kujur ${ }^{\mathrm{a}}$, D.Antony Xavier ${ }^{\mathrm{b}}$, S. Arul Amirtha Raja ${ }^{\mathrm{b}}$ \\ ${ }^{a}$ Assistant Professor, St. Joseph's College, Darjeeling, West Bengal, India \\ ${ }^{b}$ Department of Mathematics, Loyola College, Chennai, India
}

\begin{abstract}
Let $f: V(G) \rightarrow N$ be a labeling of the vertices of a graph $G$ by positive integers. Define $S(v)=$ $\sum_{u \in N(v)} f(u)$, as the sum of neighborhood of vertex $v$, where $N(v)$ denotes the open neighborhood of $v \in V$. A labeling $f$ is lucky if $S(u) \neq S(v)$ for every pair of adjacent vertices $u$ and $v$. The lucky number of a graph $G$, denoted by $\eta(G)$, is the least positive integer $k$ such that $G$ has a lucky labeling with $\{1,2, \ldots, k\}$ as the set of labels. A Lucky labeling is proper lucky labeling if the labeling $f$ is proper as well as lucky, i.e. if $u$ and $v$ are adjacent in $G$ then $f(u) \neq f(v)$ and $S(u) \neq S(v)$. The Proper Lucky number of $G$ is denoted by $\eta_{p}(G)$, is the least positive integer $k$ such that $G$ has a proper lucky labeling with $\{1,2, \ldots, k\}$ as the set of labels. In this paper we compute the lucky number $\eta(G)$ and proper lucky number $\eta_{p}(G)$ for Bloom Graph.
\end{abstract}

Keywords: Lucky labeling, Proper Lucky labeling, Bloom Graph.

\section{Introduction}

In Graph Theory vertex coloring is one of the most studied subjects. Vertex coloring is assigning the labels to the vertices, which are subject to certain constraints. Graph labeling was introduced by Rosa in 1967[1, 2]. It was further developed by Graham and Sloane in 1980[3]. The proper labeling was initiated by Karonski, Luezak and Thomason [4] Proper vertex coloring or proper labeling is coloring the vertices of a graph such that no adjacent vertices have the same color or label. Proper labeling of graph has great significance and applications in recent times [4,5,6]. It is applied in the research area of computer science especially in networking, clustering, image segmentation etc. it is also used in the field of astronomy, circuit designing, data base management, data mining, image processing, cryptography, software testing, information security. Also in the area of coding theory, x-ray crystallography, radar and missile guidance [7]. Designing of codes for radar and missile guidance is similar to labeling of complete graph in such a way that all edges are colored distinctly and the color of vertices determine the time positions at which signals are transmitted. In communication networks the labeling is used to detect the breakage of link between any two centers of broadcastings.

Lucky labeling is coloring the vertices arbitrarily such that the sum of labels of all adjacent vertices of a vertex is not equal to the sum of labels of all adjacent vertices of any vertex which is adjacent to it. Lucky labeling of graphs were studied in recent times by A. Ahai et al[8] and S. Akbari et al[9]. Lucky labeling is applied in real life situations such as transportation network, where pair wise connections are given some numerical values. And each weight could represent the stations or city with certain expenses or costs etc. They are also applicable in computational biology to model protein structures.

Proper Lucky labeling [10] is coloring the vertices such that the coloring is proper as well as lucky. The least positive integer $k$ for which a graph $G$ has a lucky labeling from the set $\{1,2, \ldots, k\}$ is called the proper lucky number of $G$, denoted by $\eta_{p}(G)$. The clique number $\omega(G)$, defined as the size of a largest clique in $\mathrm{G}$, is a lower bound for $\chi(G)$,(i.e. $) \omega(G) \leq \chi(G)[11]$.

In this paper we study lucky labeling, proper lucky labeling and compute the lucky number and proper lucky number for Bloom graph [12].

\section{Definition 2.1[5]}

\section{Lucky Labeling And Proper Lucky Labeling}

Let $f: V(G) \rightarrow \mathbb{N}$ be a labeling of the vertices of a graph $G$ by positive integers. Let $S(v)$ denote the sum of labels of the neighbors of the vertex $v$ in $G$. If $v$ is an isolated vertex of $G$ we put $S(v)=0$. A labeling $f$ is lucky if $S(u) \neq S(v)$ for every pair of adjacent vertices $u$ and $v$. The lucky number of a graph $G$, denoted by $\eta(G)$, is the least positive integer $k$ such that $G$ has a lucky labeling with $\{1,2, \ldots, k\}$ as the set of labels.

In this connection we mention the conjecture that $\eta(G) \leq \chi(G)$, for every graph $G$ [5].

Definition 2.2[10]

A Lucky labeling is proper lucky labeling if the labeling $f$ is proper as well as lucky, i.e. if $u$ and $v$ are adjacent in $G$ then $f(u) \neq f(v)$ and $S(u) \neq S(v)$. The Proper Lucky number of $G$ is denoted by $\eta_{p}(G)$, is the least positive integer $k$ such that $G$ has a proper lucky labeling with $\{1,2, \ldots, k\}$ as the set of labels.

Theorem 2.1[10]: For any connected graph $G, \eta(G) \leq \eta_{p}(G)$.

Theorem 2.2[10]: For any connected graph $G$, let $\omega$ be its clique number, then $\omega(G) \leq \eta_{p}(G)$. 
Definition 3.1

\section{Bloom graph}

The bloom graph $B_{m, n}, m, n>2$ is defined as follows:

$V\left(\left(B_{m, n}\right)=\{(x, y): 0 \leq m-1,0 \leq y \leq n-1\}\right.$ two distinct vertices $\left(x_{1}, y_{1}\right)$ and $\left(x_{1}, y_{2}\right)$ being adjacent if and only if

(i) $x_{2}=x_{1}+1$ and $y_{1}=y_{2}$

(ii) $x_{1}=x_{2}=0$ and $y_{1} \equiv y_{1}(\operatorname{modn})$

(iii) $x_{1}=x_{2}=0$ and $y_{1}+1 \equiv y_{2}(\bmod n)$

(iv) $x_{2}=x_{1}+1$ and $y_{1}+1 \equiv y_{2}(\bmod n)$

The first condition describes the vertical edges, the second and third condition describes the horizontal edges in the top most and the lower most rows respectively. Condition four describes the slant edges. Bloom graph has $m n$ vertices and $2 m n$ edges. Bloom graph is planar, tripartite and 4-regular. Grid and cylinder are planar but not regular. Whereas, torus is regular but not planar. Bloom graph is both planar and regular. The Hamiltonicity of bloom graph $B_{m, n}$ is studied in [12]. A topological structure of interconnection network should be Communication friendly. An algorithm for routing messages between any two nodes of the bloom graph $B_{m, n}$ is presented in [13]. Bloom graph $B_{m, m}$ is compared with cylinder $P_{m} X C_{m}$ which shows that bloom graph $B_{m, m}$ can achieve high performance with smaller average distance, higher network throughout, with more effective network cost and higher packing density than cylinder $P_{m} X C_{m}$.

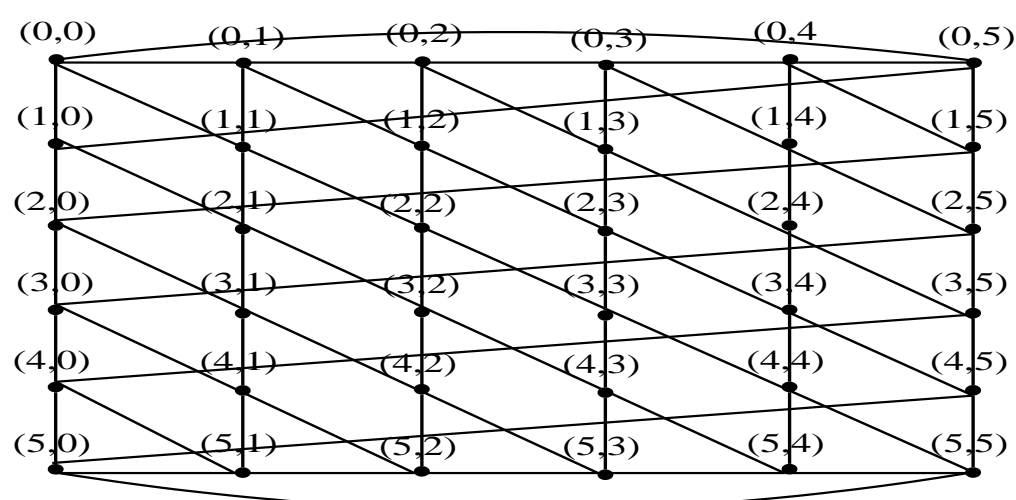

Fig. 3.1 Bloom Graph B6,6

Theorem 4.1.

IV. Lucky Labeling For Bloom Graph

The $m \times n$ dimensional bloom graph $B_{m, n}$ admits lucky labeling and $\eta\left(B_{m, n}\right)=2$, where $m>5$ and $n>3$.

Proof:

Since bloom graph $B_{m, n}$ is 4-regular $\left(B_{m, n}\right) \neq 1$.

Case 1: When $n$ is even.

Label the vertices in first row as 1 and 2 alternately, without loss of generality $(0,0)$ is assigned $1,(0,1)$ is assigned 2 and so on. Similarly label the $m^{\text {th }}$ row as 1 and 2 alternately, without loss of generality $(m-1,0)$ is assigned $1,(m-1,1)$ is assigned 2 and so on. Label the vertices in $k^{t h}$ row as 1 when $k$ is odd and as 2 when $k$ is even, $2 \leq k \leq m-1$.

Subcase $(i)$ : When $m$ is even.

The vertices in the first row will have the neighbourhood sum as 8 and 6 alternately i.e. $s(0,0)=8$, $s(0,1)=6$, and so on. All the vertices in the second row attains the neighbourhood sum as 5 i.e. $s(1, j)=$ $5,0 \leq j \leq n-1$. All the vertices in the $k^{t h}$ row attains the neighbourhood sum as 8 when $k$ is odd i.e. $s(2 i, j)=8$ when $1 \leq i \leq \frac{m-4}{2}$ and $0 \leq j \leq n-1$. All the vertices in the $k^{t h}$ row attains the neighbourhood sum as 4 when $k$ is even i.e. $s(2 i+1, j)=4$ when $1 \leq i \leq \frac{m-4}{2}$ and $0 \leq j \leq n-1$. All the vertices in the $(m-1)^{t h}$ row attains the neighbourhood sum as 7 i.e. $s(m-2, j)=7,0 \leq j \leq n-1$. All the vertices in the $m^{\text {th }}$ row will have the neighbourhood sum as 6 and 4 alternately i.e. $s(m-1,0)=6, s(m-1,1)=4$, and so on. It is evident that no two adjacent vertices have equal neighbourhood sum. (For illustration, see Figure 4.1). 


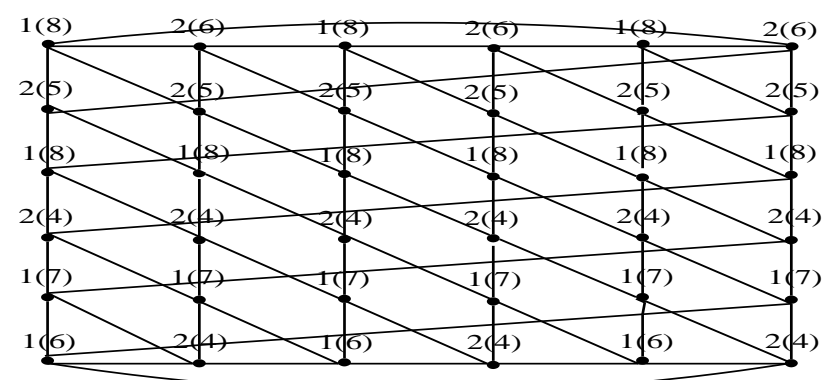

Fig. 4.1 Lucky Labeling of $\mathbf{B}_{6,6}$

Subcase (ii): When $m$ is odd.

The vertices in the first row will have the neighbourhood sum as 8 and 6alternately i.e. $s(0,0)=8$, $s(0,1)=6$, and so on. All the vertices in the second row attains the neighbourhood sum as 5 i.e. $s(1, j)=$ $5,0 \leq j \leq n-1$. All the vertices in the $k^{\text {th }}$ row attains the neighbourhood sum as 8 when $k$ is odd i.e. $s(2 i, j)=8$ when $1 \leq i \leq \frac{m-4}{2}$ and $0 \leq j \leq n-1$. All the vertices in the $k^{\text {th }}$ row attains the neighbourhood sum as 4 when $k$ is even i.e. $s(2 i+1, j)=4$ when $1 \leq i \leq \frac{m-4}{2}$ and $0 \leq j \leq n-1$. All the vertices in the $(m-1)^{t h}$ row attains the neighbourhood sum as 5 i.e. $s(m-2, j)=5,0 \leq j \leq n-1$. All the vertices in the $m^{\text {th }}$ row will have the neighbourhood sum as 8 and 6 alternately i.e. $s(m-1,0)=8, s(m-1,1)=6$, and so on. It is evident that no two adjacent vertices have equal neighbourhood sum. (For illustration, see Figure 4.2).

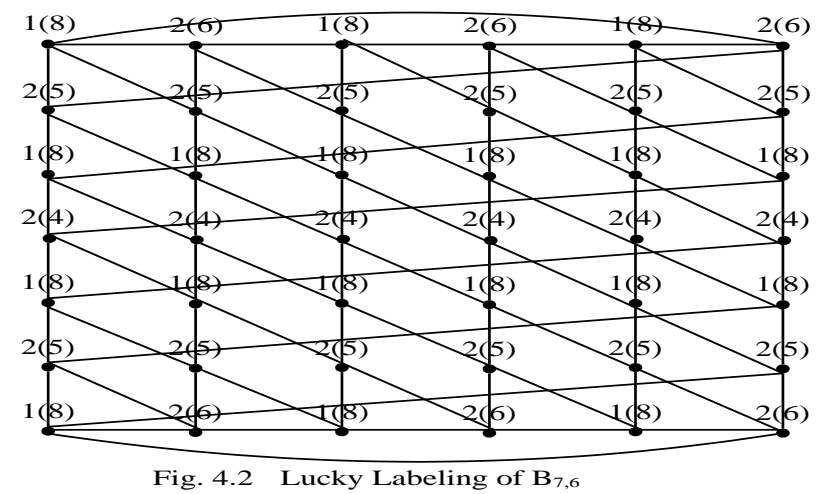

Case 2: When $n$ is odd and $m$ is odd.

Label the vertices in first row as 1 and 2 alternately, i.e. $(0,0)$ is assigned $1,(0,1)$ is assigned 2 and so on. In the second row, label the first three vertices as 2 and the rest as 1 i.e. $(1,0) \rightarrow 2,(1,1) \rightarrow 2,(1,2) \rightarrow 2$ and $(1, j) \rightarrow 1$ where $3 \leq j \leq n-1$. In the $(m-1)^{t h}$ row, label the last three vertices as 2 and the rest as 1 i.e. $(m-2, j) \rightarrow 1$ where $0 \leq j \leq n-4$ and $(m-2, n-3) \rightarrow 2,(m-2, n-2) \rightarrow 2,(m-2, n-1) \rightarrow 2$. Label the $m^{\text {th }}$ row as 1 and 2 alternately, i.e. $(m-1,0)$ is assigned $1,(m-1,1)$ is assigned 2 and so on. For the remaining vertices, label the vertices in $k^{\text {th }}$ row as 1 when $k$ is odd and as 2 when $k$ is even, $3 \leq k \leq m-2$.It is evident that no two adjacent vertices have equal neighbourhood sum.(For illustration, see Figure 4.3).

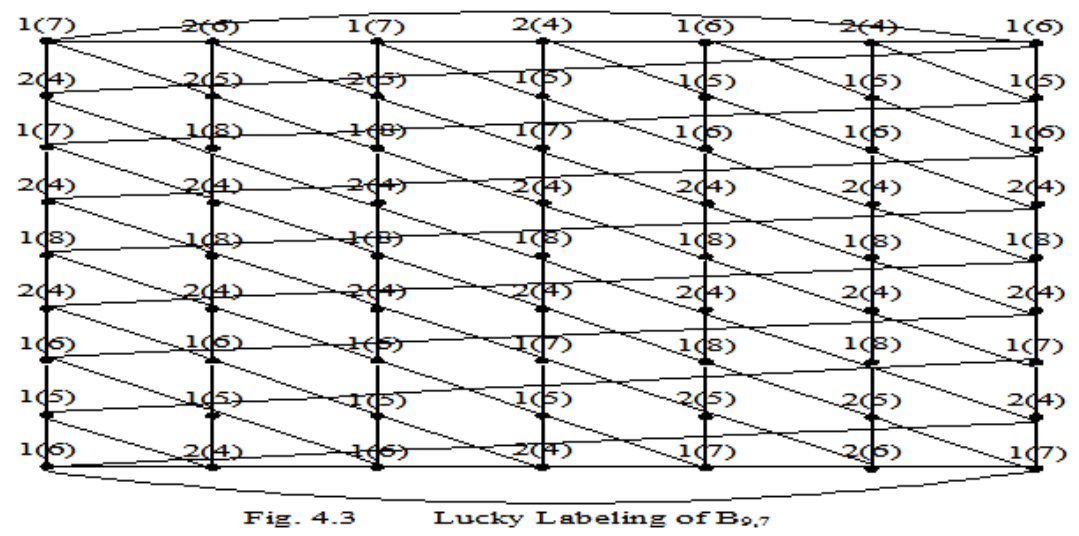


Case 3: When $n$ is odd and $m$ is even.

Label the vertices in first row as 1 and 2 alternately, i.e. $(0,0)$ is assigned $1,(0,1)$ is assigned 2 and so on. In the second row, label the first three vertices as 2 and the rest as 1 i.e. $(1,0) \rightarrow 2,(1,1) \rightarrow 2,(1,2) \rightarrow 2$ and $(1, j) \rightarrow 1$ where $3 \leq j \leq n-1$. In the $(m-1)^{t h}$ row, label the last three vertices as 1 and the rest as 2 i.e. $(m-2, j) \rightarrow 2$ where $0 \leq j \leq n-4$ and $(m-2, n-3) \rightarrow 1,(m-2, n-2) \rightarrow 1,(m-2, n-1) \rightarrow 1$. Label the $m^{\text {th }}$ row as 2 and 1 alternately, i.e. $(m-1,0)$ is assigned $2,(m-1,1)$ is assigned 1 and so on. For the remaining vertices, label the vertices in $k^{\text {th }}$ row as 1 when $k$ is odd and as 2 when $k$ is even, $3 \leq k \leq m-2$.It is evident that no two adjacent vertices have equal neighbourhood sum.(For illustration, see Figure 4.4).

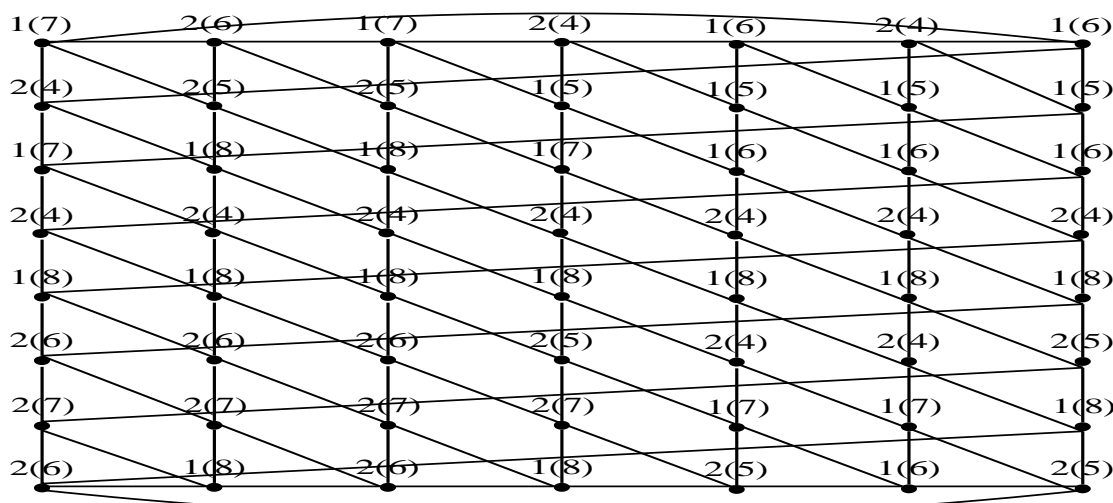

Fig. 4.4 Lucky Labeling of $\mathrm{B}_{8,7}$

Hence $B_{m, n}$ admits lucky labeling with $\eta\left(B_{m, n}\right)=2$.

Theorem 5.1.

\section{Proper Lucky Labeling For Bloom Graph}

The $m \times n$ dimensional bloom graph $B_{m, n}$ admits Proper lucky labelingand $\eta_{p}\left(B_{m, n}\right)=3$, where $m, n \geq 3$.

Proof:

Case 1: When $n$ and $m$ both are even.

Label the vertices in first row as 1 and 2 alternately, without loss of generality $(0,0)$ is assigned 1 , $(0,1)$ is assigned 2 and so on. Similarly label the $m^{\text {th }}$ row as 2 and 3 alternately, without loss of generality $(m-$ 1,0 is assigned $2, m-1,1$ is assigned 3 and so on. Label the vertices in $k t h$ row as 1 when $k$ is odd and as 3 when $k$ is even, $2 \leq k \leq m-1$. We notice that the vertices in the first row will have the neighbourhood sum as 10 and 8 alternately i.e. $s(0,0)=10, s(0,1)=8$, and so on. All the vertices in the second row attains the neighbourhood sum as 5 i.e. $s(1, j)=5,0 \leq j \leq n-1$. All the vertices in the $k^{\text {th }}$ row attains the neighbourhood sum as 12 when $k$ is odd i.e. $s(2 i, j)=12$ when $1 \leq i \leq \frac{m-4}{2}$ and $0 \leq j \leq n-1$. All the vertices in the $k^{\text {th }}$ row attains the neighbourhood sum as 4 when $k$ is even i.e. $s(2 i+1, j)=4$ when $1 \leq i \leq$ $\frac{m-4}{2}$ and $0 \leq j \leq n-1$. All the vertices in the $(m-1)^{t h}$ row attain the neighbourhood sum as 11 i.e. $s(m-$ $2, j=11,0 \leq j \leq n-1$. All the vertices in the $m t h$ row will have the neighbourhood sum as 8 and 6alternately i.e. $s(m-1,0)=8, s(m-1,1)=6$, and so on. It is evident that no two adjacent vertices have equal neighbourhood sum. (For illustration, see Figure 5.1).

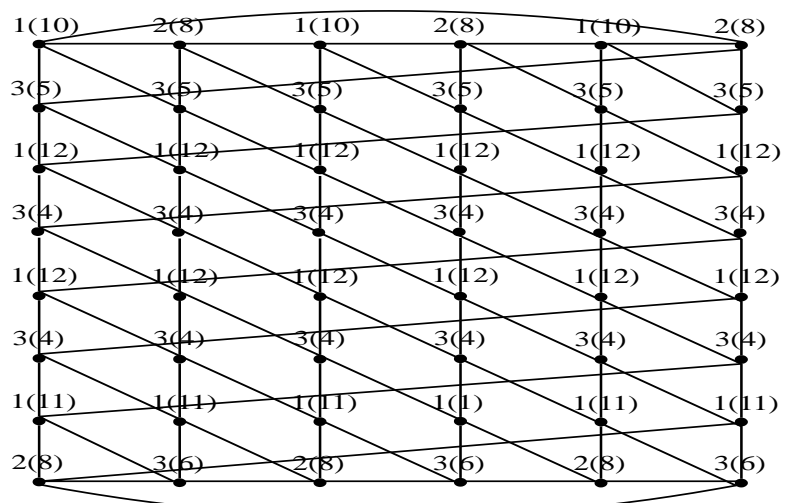

Fig. 5.1 Proper Lucky Labeling of $\mathrm{B}_{8,6}$ 
Case 2: When $n$ is even and $m$ is odd.

Label the vertices in first row as 1 and 2 alternately, without loss of generality $(0,0)$ is assigned 1 , $(0,1)$ is assigned 2 and so on. Similarly label the $m^{\text {th }}$ row as 1 and 2 alternately, without loss of generality $(m-$ 1,0 is assigned $1, m-1,1$ is assigned 2 and so on. Label the vertices in $k t h$ row as 1 when $k$ is odd and as 3 when $k$ is even, $2 \leq k \leq m-1$. It is evident that no two adjacent vertices have equal neighbourhood sum. (For illustration, see Figure 5.2).

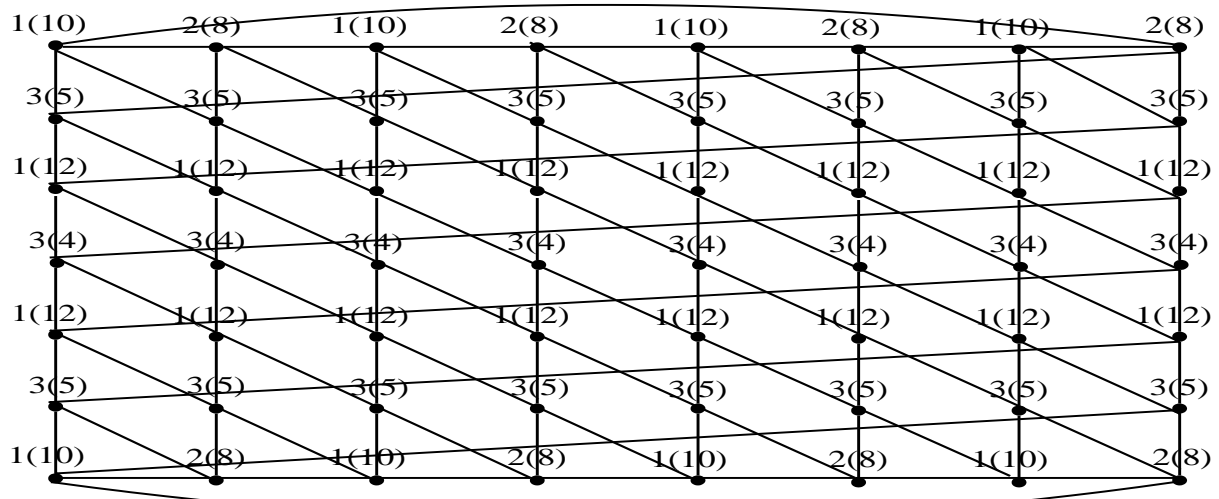

Fig.5.2 Proper Lucky Labeling of $\mathbf{B}_{7,8}$

Case 3: When $n$ is odd and $m$ is odd.

Subcase (i): when $m \bmod 3 \equiv 0$

Label the vertices in first row as 1 and 2 alternately and the $n^{\text {th }}$ column as 3 without loss of generality $(0,0)$ is assigned $1,(0,1)$ is assigned 2 and so on and $(0, n)$ is assigned 3 . Similarly label the $m^{\text {th }}$ row the first column as 3 and remaining columns as 1 and 2 alternately. Without loss of generality $(m-1,0)$ is assigned 3, $(m-1,1)$ is assigned 1 and $(m-1,2)$ is assigned 2 and so on.Label the vertices in $(3 k-1)^{\text {th }}$ row as follows: The first column is assigned as 2 and the last column is assigned as 1 and the rest of the columns as $3,1 \leq k \leq m-2$. Label the vertices in $(3 k)^{t h}$ row as follows: The first column is assigned as 3 and the last column is assigned as 2 and the rest of the columns as $1,1 \leq k \leq m-2$.Label the vertices in $(3 k+1)^{t h}$ row as follows: The first column is assigned as 1 and the last column is assigned as 3 and the rest of the columns as $2,1 \leq k \leq m-2$. It is evident that no two adjacent vertices have equal neighbourhood sum.(For illustration, see Figure 5.3).

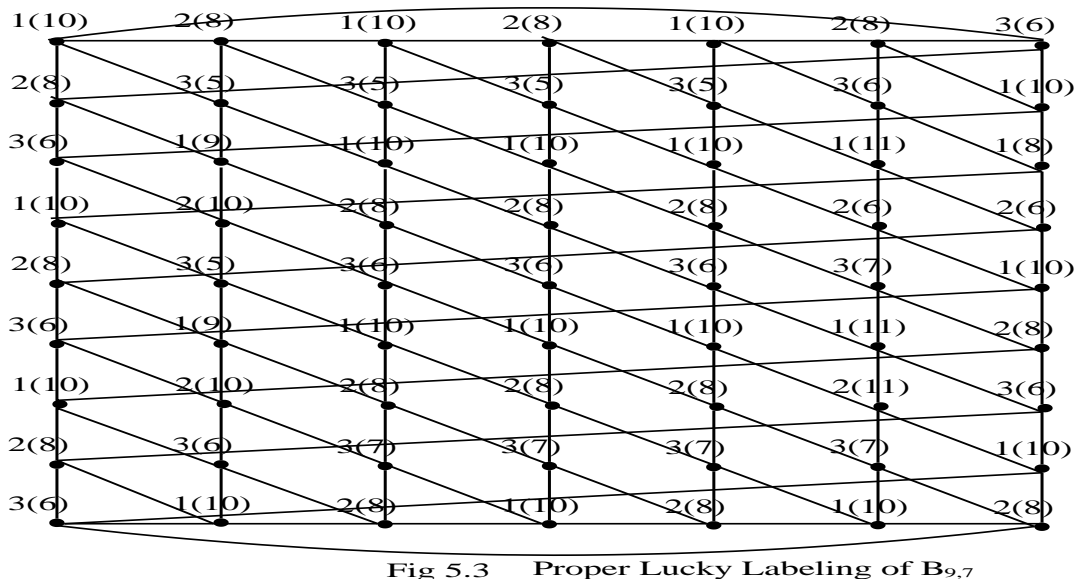

Subcase (ii): when $m$ mod $3 \equiv 1$

Label the vertices in first row as 1 and 2 alternately and the $n^{\text {th }}$ column as 3 without loss of generality $(0,0)$ is assigned $1,(0,1)$ is assigned 2 and so on and $(0, n)$ is assigned 3 . Similarly label the $m^{\text {th }}$ row the first column as 1 and remaining columns as 2 and 3 alternately. without loss of generality $(m-1,0)$ is assigned $1,(m-1,1)$ is assigned 2 and $(m-1,2)$ is assigned 3 and so on. Label the vertices in $(3 k-1)^{t h}$ row as follows: The first column is assigned as 2 and the last column is assigned as 1 and the rest of the columns as $3,1 \leq k \leq m-2$. Label the vertices in $(3 k)^{t h}$ row as follows: The first column is assigned as 3 
and the last column is assigned as 2 and the rest of the columns as $1 \quad 1 \leq k \leq m-2$.Label the vertices in $(3 k+1)^{t h}$ row as follows: The first column is assigned as 1 and the last column is assigned as 3 and the rest of the columns as $2,1 \leq k \leq m-2$.It is evident that no two adjacent vertices have equal neighbourhood sum. (For illustration, see Figure 5.4).

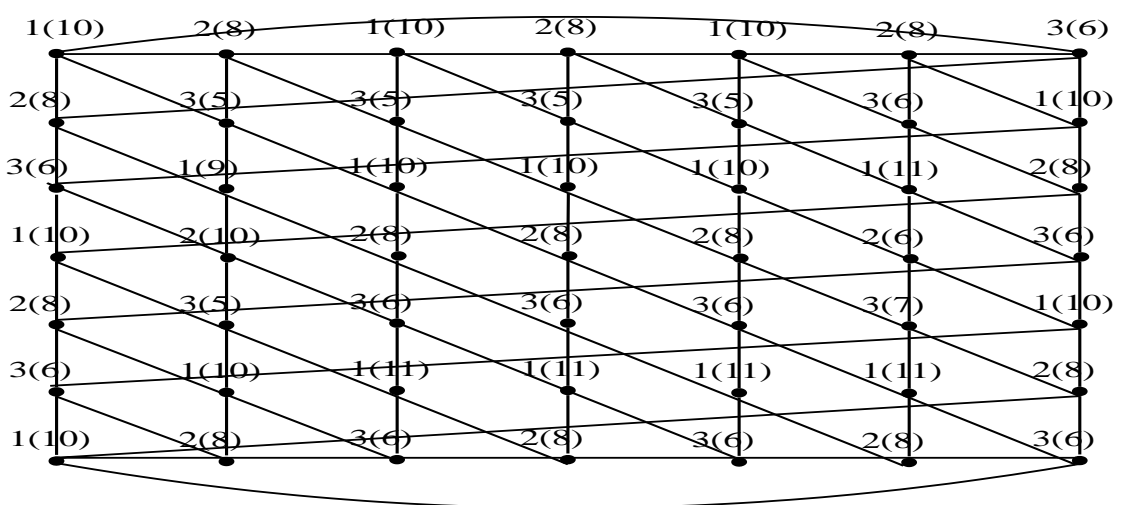

Fig. 5.4 Proper Lucky Labeling of $\mathbf{B}_{7,7}$

Subcase (iii): when $\bmod 3 \equiv 2$

Label the vertices in first row as 1 and 2 alternately and the $n^{\text {th }}$ column as 3 without loss of generality $(0,0)$ is assigned $1,(0,1)$ is assigned 2 and so on and $(0, n)$ is assigned 3 . Similarly label the $(m-1)^{\text {th }}$ row the first column as 2 second column as 3 and remaining columns as 1 and 2 alternately.without loss of generality $(m-1,0)$ is assigned $2,(m-1,1)$ is assigned 3 and rest of the column is assigned as 1 and 2 alternately. $(m-2)^{t h}$ row is assigned as follows the first column as 1 second column as 2 and the rest of the columns as 3. $(m-2)^{t h}$ row is assigned as follows the first column as 1 second column as 2 and the rest of the columns as 3.Label the vertices in $(3 k-1)^{\text {th }}$ row as follows: The first column is assigned as 2 and the last column is assigned as 1 and the rest of the columns as $3,1 \leq k \leq m-3$. Label the vertices in $(3 k)^{t h}$ row as follows: The first column is assigned as 3 and the last column is assigned as 2 and the rest of the columns as1, $1 \leq k \leq m-3$. Label the vertices in $(3 k+1)^{t h}$ row as follows: The first column is assigned as 1 and the last column is assigned as 3 and the rest of the columns as $2,1 \leq k \leq m-3$.It is evident that no two adjacent vertices have equal neighbourhood sum. (For illustration, see Figure 5.5).

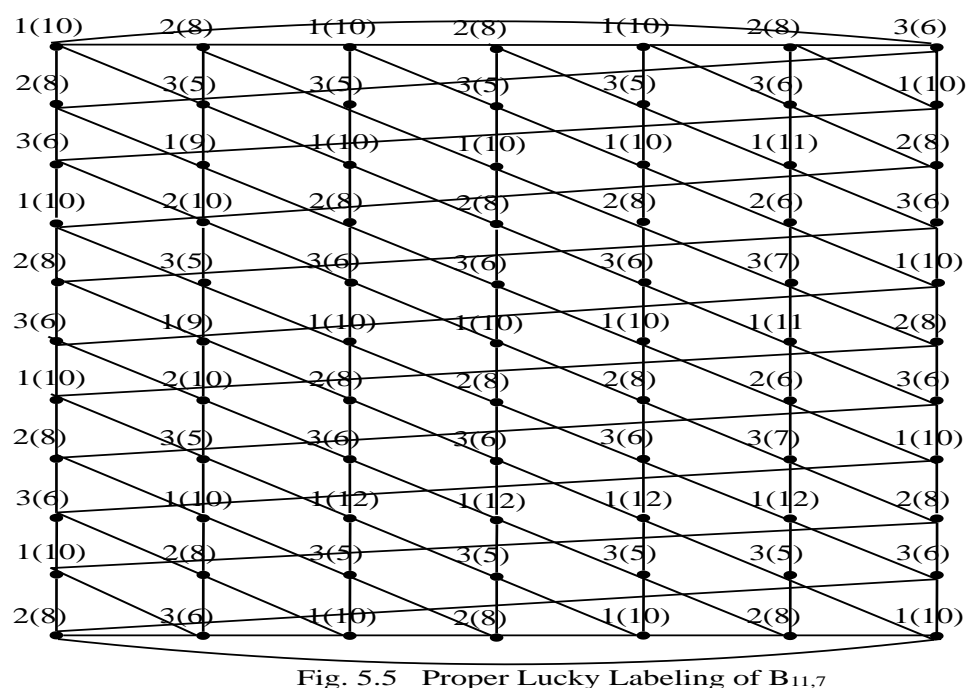

Case 4: When $n$ is odd and $m$ is even.

Subcase (i): when $\bmod 3 \equiv 0$

Label the vertices in first row as 1 and 2 alternately and the $n^{\text {th }}$ column as 3 without loss of generality $(0,0)$ is assigned $1,(0,1)$ is assigned 2 and so on and $(0, n)$ is assigned 6 . Similarly label the $m^{\text {th }}$ row the first column as 3 and remaining columns as 1 and 2 alternately. without loss of generality $(m-1,0)$ is assigned $3,(m-1,1)$ is assigned 1 and $(m-1,2)$ is assigned 2 and so on. Label the vertices in $(3 k-1)^{t h}$ 
row as follows: The first column is assigned as 2 and the last column is assigned as 1 and the rest of the columns as $3,1 \leq k \leq m-2$. Label the vertices in $(3 k)^{t h}$ row as follows: The first column is assigned as 3 and the last column is assigned as 2 and the rest of the columns as $1 \quad 1 \leq k \leq m-2$.Label the vertices in $(3 k+1)^{t h}$ row as follows: The first column is assigned as 1 and the last column is assigned as 3 and the rest of the columns as $2,1 \leq k \leq m-2$.It is evident that no two adjacent vertices have equal neighbourhood sum. (For illustration, see Figure 5.6).

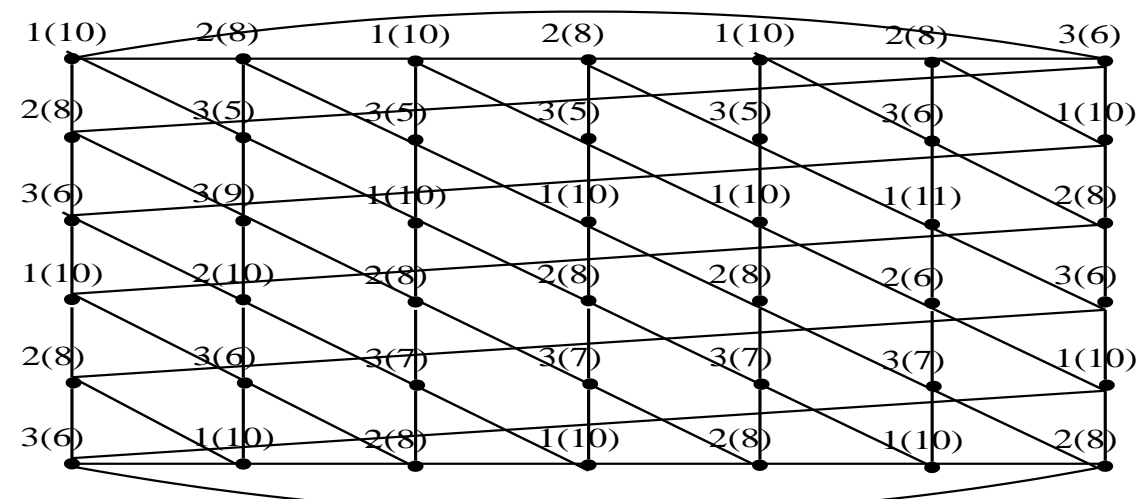

Fig. 5.6 Proper Lucky Labeling of $\mathrm{B}_{6,7}$

Subcase (ii): when $\operatorname{mmod} 3 \equiv 1$

Label the vertices in first row as 1 and 2 alternately and the $n^{\text {th }}$ column as 3 without loss of generality $(0,0)$ is assigned $1,(0,1)$ is assigned 2 and so on and $\left(0, n\right.$ is assigned 3 . Similarly label the $m^{\text {th }}$ row the first column as 1 and remaining columns as 2 and 3 alternately.without loss of generality $(m-1,0)$ is assigned $1,(m-1,1)$ is assigned 2 and $(m-1,2)$ is assigned 3 and so on. Label the vertices in $(3 k-1)^{t h}$ row as follows: The first column is assigned as 2 and the last column is assigned as 1 and the rest of the columns as $3,1 \leq k \leq m-2$.Label the vertices in $(3 k)^{t h}$ row as follows: The first column is assigned as 3 and the last column is assigned as 2 and the rest of the columns as $1,1 \leq k \leq m-2$.Label the vertices in $(3 k+$ 1)th row as follows: The first column is assigned as 1 and the last column is assigned as 3 and the rest of the columns as $2,1 \leq k \leq m-2$.It is evident that no two adjacent vertices have equal neighbourhood sum. (For illustration, see Figure 5.7).

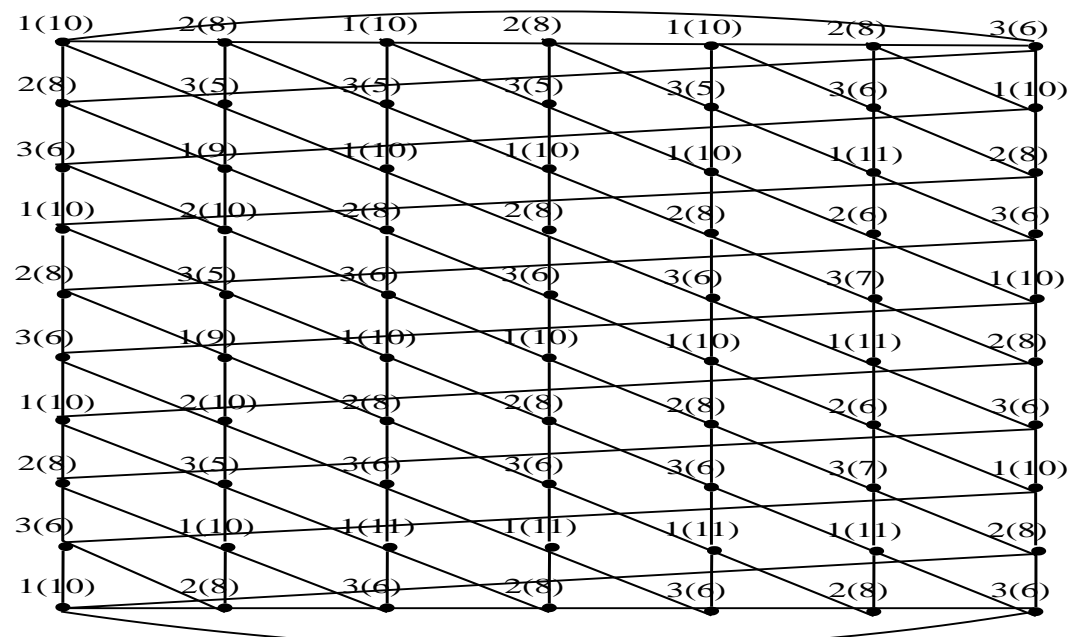

Fig. 5.7 Proper Lucky Labeling of $\mathrm{B}_{10,7}$

Subcase (iii): when $m \bmod 3 \equiv 2$

Label the vertices in first row as 1 and 2 alternately and the $n^{\text {th }}$ column as 3 without loss of generality $(0,0)$ is assigned $1,(0,1)$ is assigned 2 and so on and $(0, n)$ is assigned 3 . Similarly label the $(m-$ $1)^{\text {th }}$ row the first column as 2 second column as 3 and remaining columns as 1 and 2 alternately. without loss of generality $(m-1,0)$ is assigned $2,(m-1,1)$ is assigned 3 and rest of the column is assigned as 1 and 2 alternately. $(m-2)^{\text {th }}$ row is assigned as follows the first column as 1 second column as 2 and the rest of the columns as 3.Label the vertices in $(3 k-1)^{t h}$ row as follows: The first column is assigned as 2 and the last 
column is assigned as 1 and the rest of the columns as $3,1 \leq k \leq m-3$. Label the vertices in $(3 k)^{t h}$ row as follows: The first column is assigned as 3 and the last column is assigned as 2 and the rest of the columns as 1 , $1 \leq k \leq m-3$.Label the vertices in $(3 k+1)^{t h}$ row as follows: The first column is assigned as 1 and the last column is assigned as 3 and the rest of the columns as $2.1 \leq k \leq m-3$.It is evident that no two adjacent vertices have equal neighborhood sum.(For illustration, see Figure 5.8).

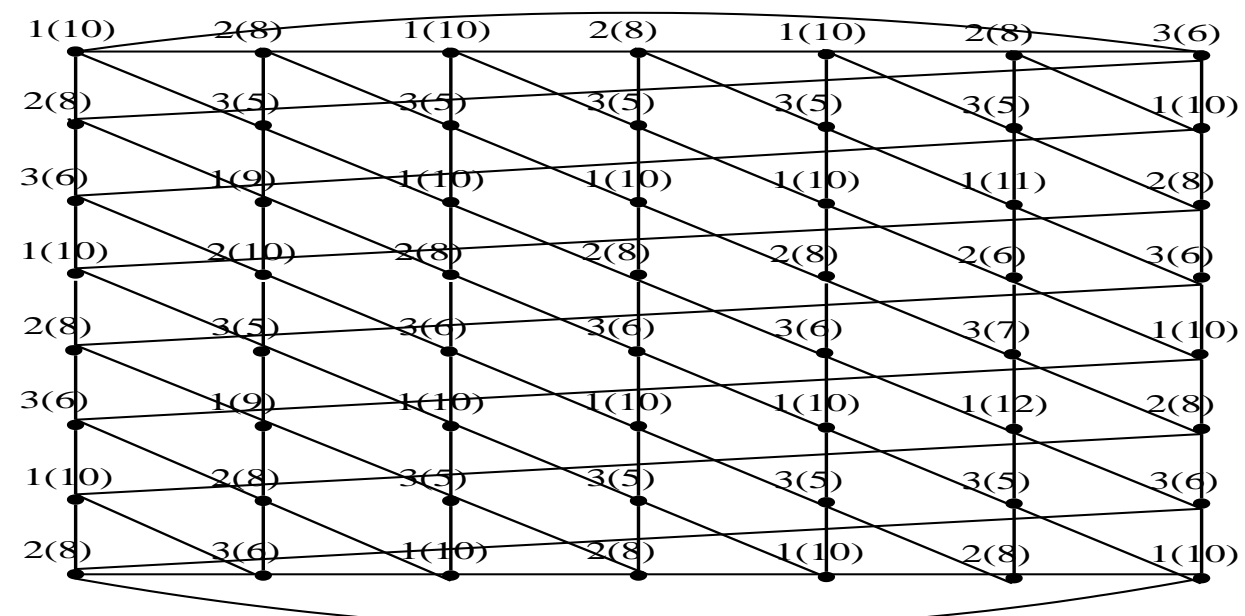

Fig.5.8 Proper Lucky Labeling of $\mathbf{B}_{8,7}$

Hence bloom graph $B_{m, n}$ admits Proper lucky labeling, $\eta_{p}\left(B_{m, n}\right)=3$.

\section{Conclusion}

In this paper we computed lucky number and proper lucky number for Bloom Graph. Further investigations are open to find proper lucky number for other interconnected networks.

\section{References}

[1]. A. Rosa, On certain valuations of the vertices of a graph, Theory of Graphs (Inter-nat. Symposium, Rome, July 1966), Gordon and Breach, N. Y. and Dunod Paris 349-355, 1967.

[2]. J. Gallian, A Dynamic survey of Graph labeling, The Electronic Journal of Combinatorics, 1996-2005.

[3]. R. L.Graham and N. J. A. Sloane, On additive bases and harmonious graphs, SIAM J. Alg. Disc. Meth, 1, 382-404, 1980.

[4]. M. Karonski, T. Luczak, A. Thomason, Edge weights and vertex colours, Journal of Combinatorial Theory, Series B, 91(1), 151$157,2004$.

[5]. S. Czerwinski, J. Grytczuk, V. Zelazny, Lucky labeling of Graphs, Information Processing Letters, 109(18), 1078-1081, 2009.

[6]. G. Chartrand, F. Okamoto, P. Zhang, The sigma chromatic number of a graph, Graphs and Combinatorics, 26(6), 755-773, 2010.

[7]. Ujwala N Deshmukh, Applications Of Graceful Graphs, International Journal Of Engineering Sciences \& Research Technology, 4.(8): Aug 2015

[8]. A. Ahai, A. Dehghan, M. Kazemi, E. Mollaahmedi, Computation of Lucky number of planar graphs in NP-hard, Information Processing Letters, vol 112, Iss 4, 109-112, 15 Feb 2012.

[9]. S. Akbari, M. Ghanbari, R. Manariyat, S. Zare, On the lucky choice number of graphs, Graphs and Combinatorics, vol 29 n.2, 157163, Mar 2013.

[10]. Kins. Yenoke, R.C. Thivyarathi, D. Anthony Xavier, Proper Lucky Number of Mesh and it's Derived architectures, Journal of Computer and Mathematical Sciences (An International Research Journal), vol. 7, Oct 2016. (inprint)

[11]. S. Held, W. Cook, E. Sewell, Safe lower bounds for graph coloring, Integer Programming and Combinatorial Optimization, 261$273,2011$.

[12]. D. Antony Xavier, C.J. Deeni, Bloom Graph, International Journal of Computing Algorithm, 521-523, Mar 2014.

[13]. C.J. Deeni, Topological Structure and Analysis of Certain Pyramid Networks, Ph. D thesis, University of Madras, , 111-113, 2015. 